\title{
Application of infographic modeling anthropotechnics in management and optimization of production programs
}

\author{
Alexey Yurgaytis ${ }^{1, *}$, and Vitaly Chulkov ${ }^{1}$ \\ ${ }^{1}$ Moscow State University of Civil Engineering, 129337, 26, Yaroslavskoye Shosse, Moscow, Russia
}

\begin{abstract}
This article discusses possible applications for modeling the infographic system in terms of planning in general and developing programs of construction organizations. The authors present the basic prerequisites and principles for creating an integrated system of organizational and technological design, taking into account infographic approaches. As a result of research, analysis of anthropotechnical approaches to the formation of a safe production environment, the developed interdisciplinary system-technical activity on the use of innovative "screens" is described, which requires the identification of its systematizations, relationships and limitations.
\end{abstract}

\section{Introduction}

Life safety is a new scientific and practical area, the roots of which go back into the millennia. In the struggle for their food security, man mastered or created agriculture, hunting, crafts, science and technology. Technical civilization is developing at a rapid pace and is changing the environment to such an extent that the skills and mechanisms of adaptation of man and many other biological objects to the environment, acquired over the millennia, have ceased to work or have been lost.

Artificial habitat and activities - buildings and structures, transport, artificial materials, technical means, etc. dramatically changed the safety of human activity and life. We agree to understand life safety (LS) as a scientific and practical area in which educational, technical, anthropotechnical and organizational means of ensuring the possibility of continuing life are developed.

Ecology was initially defined as a scientific and practical discipline on the interaction of man and the environment and was obliged to provide LS of human with special technologies and systems of organization [1]. This position required the creation of logic and models of presentation of the subject of research. Ecology could become one of the rapidly developing areas of interdisciplinary research and a point of growth for the emergence of new interaction logics and technologies. However, representatives of environmental science have tried to take advantage of the existing baggage of logics and representations of the scientific subject.

This has led to the fact that the interdisciplinary field of ecology has gradually turned into an independent area, using the usual schemes of "subject-object", which do not allow solving a number of logical contradictions of humanitarian, technical and vital approaches in science and

*Corresponding author: aljurgaitis@gmail.com 
practice. Ecology has failed to build logics and models that take into account the existence of these three approaches simultaneously, to link them with each other, to positively remove the logical conflicts and contradictions existing between them, that is, it has failed to build common logics and models of the interaction of Life, Technical and the Environment [2, 3, etc.].

\section{Materials and methods}

Ecology has become the science of the movement of waste activities and human life, in the social practice of helpless attempts to reduce the pathogenic impact of these wastes on the environment and man.

A scientific discipline gets a strong impetus in its development if it allows to solve a problem situation or a conflict arising at the junction of approaches. Conversely, if the conflict in this area is removed, or the representatives of this scientific discipline for one reason or another were eliminated from its resolution, the scientific direction loses the pace of its development, relevance and meaningfulness of the results.

Ecology could not work out the issues of ensuring human life safety in the technogenic and natural environment. Gradually, ecology accumulates a fundamental amount of knowledge about natural nature and, to a lesser extent, about the artificial environment. However, the issues of ensuring life safety for a particular person in the environment where he is forced to carry out activities have fallen out of the consideration of ecology.

The separation of life safety into a separate scientific and practical direction is a natural phenomenon. The mechanisms of human adaptation to new conditions do not always work and the technogenic environment often carries more danger and potential harm than the possibility of providing comfort and efficiency of staying in it.

The use of artificial materials in the construction and decoration of housing has led to significant chemical pollution of the human environment. The use of chemical fertilizers in agriculture, hormonal drugs in cattle and poultry has made food potentially dangerous. The increasingly widespread introduction of transgenic foods has not been tested for long-term effects at all, and in fact the experiment is being conducted on the populations of entire States. The threat of life and the possibility of its elimination have become a separate extremely capacious subject, requiring the use of the power of modern scientific approach.

Today, the LS collects empirical material, develops technical means and educational programs designed to ensure a safer human existence, reduce the risk of death and injury in situations beyond environmental norms.

Effective development of technologies and means of life safety cannot do without changing the approach to research and design of construction production and construction facilities, without changing the basic methodological schemes of LS construction as a scientific and practical area.

Domestic and foreign scientific schools, individual scientists and researchers, design and construction organizations pay considerable attention to the problem of life safety. Life safety issues are used in the design and construction of comfortable and affordable housing of the new generation. Bay windows, dormers with windows, a combination of white, yellow and red brick give the houses a unique look. There are known attempts to create a harmonious dwelling environment that meets the needs of a person, improved space planning and town planning solutions, and the development of new construction methods.

The scientific school of the Moscow state construction University (Akhremenkov P. A., Belov Y. A., Druzhinin A. I., Zabegaev A.V., Konstantinov V. F., Kotlyarevsky V. A., Kochetkov K. E., Matkaev G. I., Nosach A. A., Panchenko A. A., Cherepanov E. N., Chernodubov Y. F. and many others) is authoritative and known for its success in studying the problem of life safety and developing methods for solving its problems.

Engineering and medicine within the framework of life safety conduct research on representative samples that provide statistical reliability of the results and the formation of 
standards on their basis. Industrial and agricultural products produced according to these standards, in the best case can be safe for "fully consistent with the average indicators", that is, "healthy" person, has not yet been exposed to this very product. But each of us is sick and healthy in his own way, uses his own special mechanisms of adaptation to loads or their compensation, has a characteristic "safety margin" and a specific history of obvious or not obvious overloads, felt or not felt by a person.

This is a consequence of the difference not only people, but also technically created environments of their habitat. Combinations of factors of such environment are so various that one premise from another, even neighboring, from the point of view of life safety of the concrete person, can differ cardinally.

During the first two decades of the new Millennium, a new scientific and practical direction construction anthropotechnics (CA), as a result of philosophical, logical and methodological studies of the phenomenon of development of the dialectic system "man - technology environment (MTE)".

CA uses the system approach in relation to the description of activity in the system of MTE, the theory of functional systems (Anokhin P. K.), the theory of "big systems" (Buslenko N. P.), the system engineering of construction (Gusakov A. A.), infography (Chulkov V. O.), combining elements of artificial and natural approaches in the distinctions of Shchedrovitsky G. P. and his methodical movement $[4,5,6$, etc. $]$.

CA investigates a person (biological object, individual, social group), the external environment of technical objects and means (technology) formed by him, as well as the surrounding natural environment of habitat and activity, that is, the MTE system in all interactions of a person, technology and environment. The CA is designed to contribute to ensuring the necessary level of life safety and the level of human comfort in the MTE system, preserving and/or improving the quality and volume of health of the person who consumes construction products, produces it or participates in the construction processes.

The main differences of CA are:

- natural attitude to the environment and artificial-technical attitude to the physiology and self-organization of a person or social groups;

- association of technical, humanistic and aesthetic areas of knowledge and activities to meet human needs;

- use, in addition to the classical theoretical and logical and game tools, instrument and hardware components that provide inspection, diagnosis and monitoring of the MTE system as a whole and its individual elements;

- use of infographic methods, tools and models for modeling activities in CA, display and analysis of intermediate and final results [7, etc.].

CA is developing a model of multi-point logic of parameter space in which:

1) implements the dialectical "unity and struggle of opposites" of the three components of the MTE system (man, technology and environment);

2) implement simulated controlled development of this system;

3) investigates and designs the control mechanisms of the system MTE and dyads "M-T", "M-E" and "T-E";

4) constructs in society "machines from people" and manages them;

5) develops individual techniques of self-organization of its life safety in society;

6) modifies the MTE system in order to maximize the approximation of its parameters to the capabilities of a particular person living in it;

7) carries out preparation of the concrete person to life safety in the conditions of concrete system of MTE;

8) prepares a person for the state when he himself is forced to change (transform, adapt) the MTE system to ensure the safety of his life. 
Docking of scientific and practical directions relating to human life safety takes place in CA on a single field of construction ecology.

Construction anthropotechnics of life safety (CA LS), as an application of CA in the field of LS, uses known or newly developed in engineering, ecology, organizational management, psychology, biology and medicine methods of examination, diagnosis, monitoring, adaptation and compensation of unilateral or mutual pathogenic effects of components of the MTE system.

However, the application of these methods within the framework of the CA LS is not based on typologies and systematizations obtained by anthropologists, physicians, engineers and other scientists individually on different people and in different anthropogenic or natural environment. CA LS conducts direct research and monitoring of the interaction of a particular person, a particular technology and the environment (that is, a specific unit system of MTE).

The regularities thus detected, built up by the typology and systematization of the properties of a particular person and a particular environment, in contrast to those practiced so far in the normative approach, become the basis for the normalization of a particular environment and a particular person.

Phenomenological analysis (PA), that is, the identification and study of many ideal and real "layers" of the functioning of a complex MTE system, develop explicitly since the beginning of theXX century (Hartman N., [8], Husserl G. A. E., [9], Ingarden R. V., [10], Starostin B. A. [11], etc.). The studied "layers" of the PA of the MTE system (physical, psychological; norms and laws) can be supplemented with new "layers" as the boundaries of cognition or reasoned necessity expand (Popper K. R., [12], etc.).

The main component and the main argument for the functioning of the MTE system, as well as an indispensable component of all these "layers" of PA is a person.

In process of development of representations about system of MTE, the person suggested to consider:

- as separate "subject region of life" (Husserl G. A. E., 1913-1929);

- as the transcendent subject which is not reduced to any of the listed "layers" (Hartman N., 1979);

- as new separate "layer" PA of systems of MTE (Chulkov V. O., 1997, “anthropotechnical approach"), etc.

As part of the anthropotechnical approach, CA acts as an independent direction of research and design and experimental engineering activities in the MTE system, the typical representative of which is the construction industry.

Anthropotechnical approach allows:

- to form individual and collective recreational techniques;

- to provide prevention of diseases and overloads in the workplace or at the home;

- to manage the level of comfort of living in a particular environment for a particular person;

- to effectively develop post-industrial technologies in the real information sector of the economy.

Life safety, as a direction of interdisciplinary research and development of complex technologies, is becoming an increasingly active point of a new round of growth of scientific and technical advance.

Any field of activity using infographic "objects" and "processes" [13] can be considered and, as a rule, professionals of different specialization (engineers, managers, economists, medics, etc.) as their object of research (OR) on their separate "screen" S1i or S1j (figure 1). 


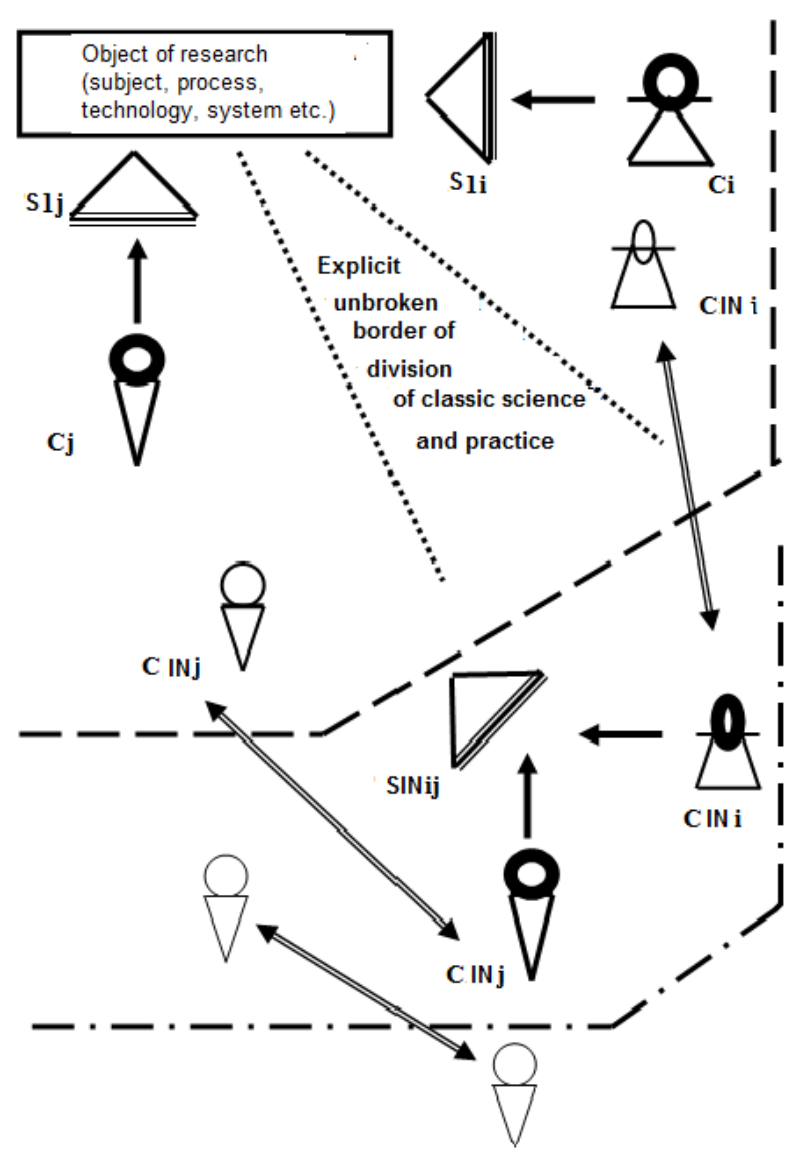

Fig. 1. Formation of interdisciplinary knowledge in functional subsystems of life safety [14].

\section{Results and discussion}

The term "screen" in the proposed interpretation is widely used by Shchedrovitsky G. P. in the theory of activity and organizational-activity games and Kotelnikov S. I. in the theory of layout design in CAD.

Specialists $\mathrm{Ci}$ and $\mathrm{Cj}$ occupy the corresponding "screens" $\mathrm{S} 1 \mathrm{i}$ and $\mathrm{S} 1 \mathrm{j}$ formally fixed professional "positions" in which they create or master the information of their separate specialization (for example, developers or users of CAD) in relation to OR.

In a particular type of activity, the professional is distinguished from the layman by the knowledge of all positions, their respective systematizations of knowledge and technologies of activity, the relationships between all positions and the limitations of each position. The listed set of components characterizes "culture" of activity.

Recall that even in the days of the Roman Empire, the concept of culture was defined as an invisible transparent wall that does not allow a barbarian to become a Roman.

The culture of activity should be inherent in all certified specialists of a particular type of activity and are evidence of their professional qualifications. If this activity is recognized by the community of specialists or society as a whole, has a confirmation of the reliability of the results obtained, and information technologies of such activity are in demand, then usually such scientific or practical activity is called "classical". 
The set of specific characteristics of the activity in the chosen professional field and the existing restrictions in it, outlined in figure 1 with dashed line, highlights and identifies the current time "classical" science or practice.

Specialists $\mathrm{Ci}$ and $\mathrm{Cj}$, located within this "border", as a rule, are orthodox and "refined", proud of the self-isolation of their field of activity, do not "confuse" with other areas of science and practice, like to point out mistakes and teach others. They seem to "rest their eyes" only on their "screen" and do not want to look at other famous screens. However, always in the environment of any activity there are not only "orthodox", but also "innovators" (not to be confused with crooks, hack, not-qualified and not mastered the "culture of the orthodox" people !!).

"Innovators", having mastered the culture of "orthodox", tend to contact the actions of other "screens" (in contrast to the "orthodox") and are looking for opportunities to create common with them innovative "screens" SINij, not yet known in a particular "classical" field of science or practice.

Interdisciplinary system-technical activity on the use of innovative "screens" requires the identification of their systematization, relationships and limitations [15]. The set of such new constraints shown in figure 1 with dash-dotted line, expands and concretizes the previously existing area of knowledge (which is located inside the dotted line in figure 1).

Over time, this innovative activity, having been tested for the reliability, quality and stability of the provided scientific and practical results, can become part of the classical sciences and practices as an equivalent component.

The appearance in figure 1. beyond the dashed "border" indicates that the number of innovative experiments and new "screens" expanding consistently beyond the "class" may be finite. It is limited only by "common sense" and the needs of society.

\section{Conclusion}

Thus, the research once again confirms the assumption Kotov I. I. that one of the promising areas of CAD development should be "secondary modeling", understood as the creation of "models of models".

\section{References}

1. P. Oleinik, A. Yurgaytis, MATEC Web of Conferences 117, 00130 (2017) https://doi.org/10.1051/matecconf/201711700130

2. V.O. Chulkov, I.Y. Masturov, S.K. Juraev, Internet: news and review 2(2-1), 79-82 (2002)

3. V.O. Chulkov, I.Y. Masturov, S.K. Juraev, Intersectoral information service 1, 41-43 (2003)

4. Development and implementation of automated systems in design (theory and methodology) (Central research and design and experimental Institute of automated systems in construction, Moscow, 1975)

5. G.P. Shchedrovitsky, Izvestiya 234 (1961)

6. G.P. Shchedrovitsky, Scientific notes of Tomsk University: Collection 41, $81-92$ (1962)

7. V.O. Chulkov, Infography.- In the book.: System engineering (Fund "New Millennium", Moscow, 2002)

8. N. Hartman, Foundation of ontology (Science, St-Petersburg, 2003)

9. G.A. Husserl, Questions of philosophy 7, 136-176 (1992)

10. R.V. Ingarden, Studies in aesthetics (Publish house of foreign literature, Moscow, 1962)

11. B.A. Starostin, Parameters of the development of science (Nauka, Moscow, 1980) 
12. K.R. Popper, Open society and its enemies (Phoenix, international Fund "Cultural initiative", Moscow, 1992)

13. V.O. Chulkov, Methodical recommendations on complex processing of documentation (system technical problems) (Central research and design and experimental Institute Proekt, Moscow, 1983)

14. O.V. Chulkov, Life Safety. Organizational and anthropological-technical reliability of the functional systems of the mobile environment of construction industry. Series "Infographic fundamentals of functional systems" (Publishing house ASV, Moscow, 2003)

15. V.O. Chulkov et al., Internet: news and review 2 (2-1), 87-90 (2002) 